\title{
"Biopharmacy is Back!"* Meeting Report: The Role of the Biopharmaceutics Classification System and In Vitro-In Vivo Correlations in the Approval of Oral Drug Products
}

I n 1997, a workshop on the "Scientific Basis for and Applications of the Biopharmaceutics Classification System and In Vitro-In Vivo Correlations" was held in Washington DC under the auspices of CRS, AAPS and the FDA. A European version of this workshop, this time in cooperation with EUFEPS and APV (the International Organization for Pharmaceutical Technology) as well as CRS and the FDA, was held March 25-27 this year in Frankfurt, Germany.

The three day workshop featured sessions on various aspects of the Biopharmaceutics Classification Scheme. Professor Henning Blume of the German Central Laboratory (ZL) and Dr. Roger Williams of the FDA kicked off the workshop with an overview of international bioequivalence standards and introduction to regulatory standards for drug product quality from the standpoint of the FDA.

The following three sessions were devoted to the basic building stones of the BCS, namely gastrointestinal variables, membrane permeability, and solubility and drug dissolution. Of particular interest in terms of gastrointestinal variables were the data presented by Prof. Clive Wilson from the University of Strathclyde concerning diurnal effects on the movement of dosage forms into and through the colon. Dr. Jürgen Stein, from the University of Frankfurt, discussed alterations in drug uptake associated with disease states, using inflammatory bowel disease, resected small bowel patients and pancreatitis as case examples.

The presentations on permeability covered the whole spectrum of predictive and experimental techniques, from theoretical predictions based on physical chemical data (Dr. van de Waterbeemd, Pfizer, Inc.) through to a presentation on how the various methods can be integrated into a practical approach to preclinical screening in the pharmaceutical industry (Dr. Phil Smith, SmithKline Beecham). A particularly important point that was brought out by Prof. Per Artursson (University of Uppsala) and highlighted in the pursuant discussion by Dr. Ajaz Hussain (FDA) was the need for reference compounds when using cell cultures for permeability

${ }^{*}$ quote from Prof. Henning Kristensen studies, since results can vary widely between laboratories. The administration of a 'cocktail' of several new compounds in whole animal studies to assess their permeabilities was presented by Dr. Phil Smith as a way of reducing the number of studies needed to sort out candidates for oral delivery.

In the session on solubility and dissolution, Prof. Gordon Amidon (University of Michigan) laid the groundrules for determining whether a substance fits the BCS criteria for highly soluble or not. Prof. Jennifer Dressman (University of Frankfurt) followed with a discussion of how to establish dissolution conditions that are more representative of those in the GI tract. Main areas of concern remain the simulation of fed state conditions in the stomach and trying to reproduce the wide-ranging hydrodynamic conditions in the upper GI tract. Dr. Martin Siewert (Hoechst AG) underscored the need to develop dissolution tests that can be used both as a quality control mechanism and as a measure of bioequivalence. To round off the session, Dr. Bertil Abrahamsson (Astra Hässle, Sweden) presented several illuminating case examples for successful as well as unsuccessful attempts to create dissolution models for the bioavailability of ER products.

Next followed a discussion of various appropriate data analysis techniques. Prof. Christos Reppas (University of Athens) gave a detailed explanation of how the $\mathrm{f} 2$ (similarity) factor functions and discussed how the sampling in in vitro studies must be set up in order to assure meaningful comparisons of dissolution profiles. Dr. Dierk Brockmeier (Hoechst AG) followed with a presentation highlighting the clinical side of the in vitro/in vivo coin, discussing both convolution and deconvolution techniques. Dr. Johannes Krämer (ZL) then presented practical examples for the correlation of in vitro and in vivo data using case studies performed at $Z \mathrm{~L}$.

On the third day, the emphasis moved to regulatory aspects and the impact of the guidance process. After an introductory overview to the FDA's regulatory policies for oral formulations from Dr. Larry Lesko, the Guidances for IR, ER and the BCS were summarized individually by Dr. Vinod Shah, Dr. Hank Malinowski and Dr. Ajaz Hussain. In these

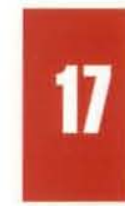




\section{“Biopharmacy is Back!”...continued}

presentations the FDA showed its willingness to consider adjustments to the Guidelines. For example, Dr. Hussain indicated that the requirement for High Permeability could probably be loosened to allow compounds with permeabilities corresponding to $80 \%$ absorption or better to be admitted to this category. It was also brought out that the Guidelines are meant to ease the stringency of requirements for bioequivalence studies, so that certain preparations (especially those IR products containing class I compounds and ER products with good in vitro/in vivo correlation histories) could be exempted from bioequivalence studies.

The workshop then deviated from its American precursor, in that the last session was devoted to international perspectives and the outlook for harmonization of regulatory policies. In response to the FDA's BCS philosophy in Europe (Dr. Roger Williams, FDA) representatives from the EMEA (Dr. Panayiota Bouka), WHO (Dr. Sabine KoppKubel), BfarM (Dr. J. Limberg), the European Pharmacopeia (Prof. Henning Kristensen) and the German pharmaceutical industry (Dr. Gertrud Ahr) gave their perspectives on the regulatory climate in Europe and where the chances for harmonization and important diversities in opinion lay. All representatives were in agreement that a harmonization of policy is urgently needed, so that the industry is not overburdened with differences in regulatory approaches that result in bioequivalence studies having to be run on a country-to-country basis. Dr. Kopp-Kubel stressed that the need for substitutes for bioequivalence studies is particularly urgent in the developing nations. An interesting difference between the German authority's (BfarM's) approach and the FDA approach is that the BfarM takes into consideration the therapeutic category of the drug substance when deciding whether bioequivalence studies have to be run or not. Therapeutic categories in which an over/underdosage due to enhanced/diminished bioavailability could lead to grave consequences for the patient are in general required to undergo bioequivalence testing. Other categories may receive exemptions. As a further discussion point, Dr. Ahr (Bayer AG) introduced the concept that drugs with fast absorption and elimination rates are more susceptible to bioinequivalence than those with long halflives. Thus, from the European perspective, it seems that in addition to the BCS criteria of solubility and permeability, additional factors such as therapeutic category and pharmacokinetics can also play an important role in the decision as to whether bioequivalence studies are required. It will be interesting to see if and how the FDA integrates these additional concepts into its thinking, and conversely, to what degree the European authorities will adopt the BCS concept into their policies. One thing is sure, the 150 plus participants from 12 different countries all came away from the workshop with a better understanding of the issues involved in and the approaches currently being taken in regard to the regulation of bioequivalence. 